\title{
Relationships between hand-grip strength, socioeconomic status, and depressive symptoms in community-dwelling older adults
}

\author{
Kyu-Man Han ${ }^{1}$, Jisoon Chang², Ho-Kyoung Yoon ${ }^{1}$, Young-Hoon Ko', Byung-Joo Ham¹, \\ Yong-Ku Kim¹, Changsu Han ${ }^{1, *}$
}

1 Department of Psychiatry, Korea University College of Medicine, Seoul, Republic of Korea; 2 Department of Psychiatry, Seoul Metropolitan Eunpyeong Hospital, Seoul, Republic of Korea. *Corresponding author: Changsu Han (hancs@korea.ac.kr)

Background: Hand-grip strength (HGS) is a valid and simple assessment technique to measure muscle strength in clinical practice. Low HGS is associated with increased mortality, reduced health-related QOL, accelerated dependency in activity of daily living, cognitive decline, depressive symptoms in older populations. We aimed to investigate the potential moderating effect of SES on the association between HGS and depressive symptoms and the potential mediating effect of HGS on the association between SES and depressive symptoms using a nationally representative sample of older adults.

Table 1. Associations of hand-grip strength with depressive symptoms and suicidal ideation in a logistic regression analysis.

\begin{tabular}{ccccc}
\hline \multirow{2}{*}{$\begin{array}{c}\text { HGS } \\
\text { (tertile) }\end{array}$} & \multicolumn{2}{c}{ Depressive symptoms } & \multicolumn{2}{c}{ Suicidal ideation } \\
\cline { 2 - 5 } & OR & $95 \%$ CI & OR & $95 \%$ CI \\
\hline Lowest & $\mathbf{1 . 8 5}$ & $1.25-2.74$ & $\mathbf{1 . 8 5}$ & $1.31-2.62$ \\
Middle & 1.36 & $0.92-2.01$ & $\mathbf{1 . 5 0}$ & $1.07-2.11$ \\
Highest & 1 & & 1 & \\
\hline
\end{tabular}

Table 2. Post-hoc multiple regression analysis investigating the correlation between HGS and PHQ-9 score in subgroups determined by income level.

\begin{tabular}{|c|c|c|c|c|}
\hline \multirow{2}{*}{ Variables } & \multicolumn{4}{|c|}{ PHQ-9 score } \\
\hline & B & SE & Beta & $\mathbf{p}$ \\
\hline \multicolumn{5}{|c|}{$<$ Lower income level $>$} \\
\hline HGS $(\mathrm{kg})$ & -0.084 & 0.018 & -0.162 & $<0.001$ \\
\hline $\mathrm{R}^{2}$ (adjusted) & \multicolumn{4}{|c|}{0.098} \\
\hline \multicolumn{5}{|c|}{$<$ Higher income level $>$} \\
\hline HGS $(\mathrm{kg})$ & -0.036 & 0.017 & -0.119 & 0.036 \\
\hline $\mathrm{R}^{2}$ (adjusted) & \multicolumn{4}{|c|}{0.066} \\
\hline
\end{tabular}

Methods: Data were analyzed from 3,619 community-dwelling adults aged 60 years or older from the Korea National Health and Nutrition Examination Survey (KNHANES) conducted in 2014 and 2016. HGS was measured using a digital hand-grip dynamometer. Depressive symptoms were evaluated using the 9-item version of the Patient Health Questionnaire-9 (PHQ-9). SES was assessed using equivalent monthly household income and education level. $A$ series of hierarchical moderated regression analyses were performed with respect to the interactive effects of HGS and SES on depressive symptoms in older adults. We investigated the potential mediation effect of HGS on the association between SES and depressive symptoms using mediation analysis by Hayes and Preacher (2014). All socioeconomic and health-related factors were included as covariates in the analyses (age, sex, education, income, marital status, economic activity, residential region, BMI, number of chronic disease, alcohol, smoking, physical activity).
Results: Older adults in the lowest tertile of HGS measures were more likely to have experienced depressive symptoms compared to those in the highest tertile (odds ratio=1.95, 95\% confidence interval=1.25-2.74). A significant moderating effect of household income level was observed on the association between HGS and $P H Q-9$ score $(P=0.014)$. Older adults with a low income had a stronger inverse correlation between HGS and PHQ-9 score compared to those with a high income (low income: beta $=-0.162, P<0.001$; high income: beta $=-0.119$, $\mathrm{P}=0.036$ ). HGS partially mediated the association between low income and depressive symptoms.

A.

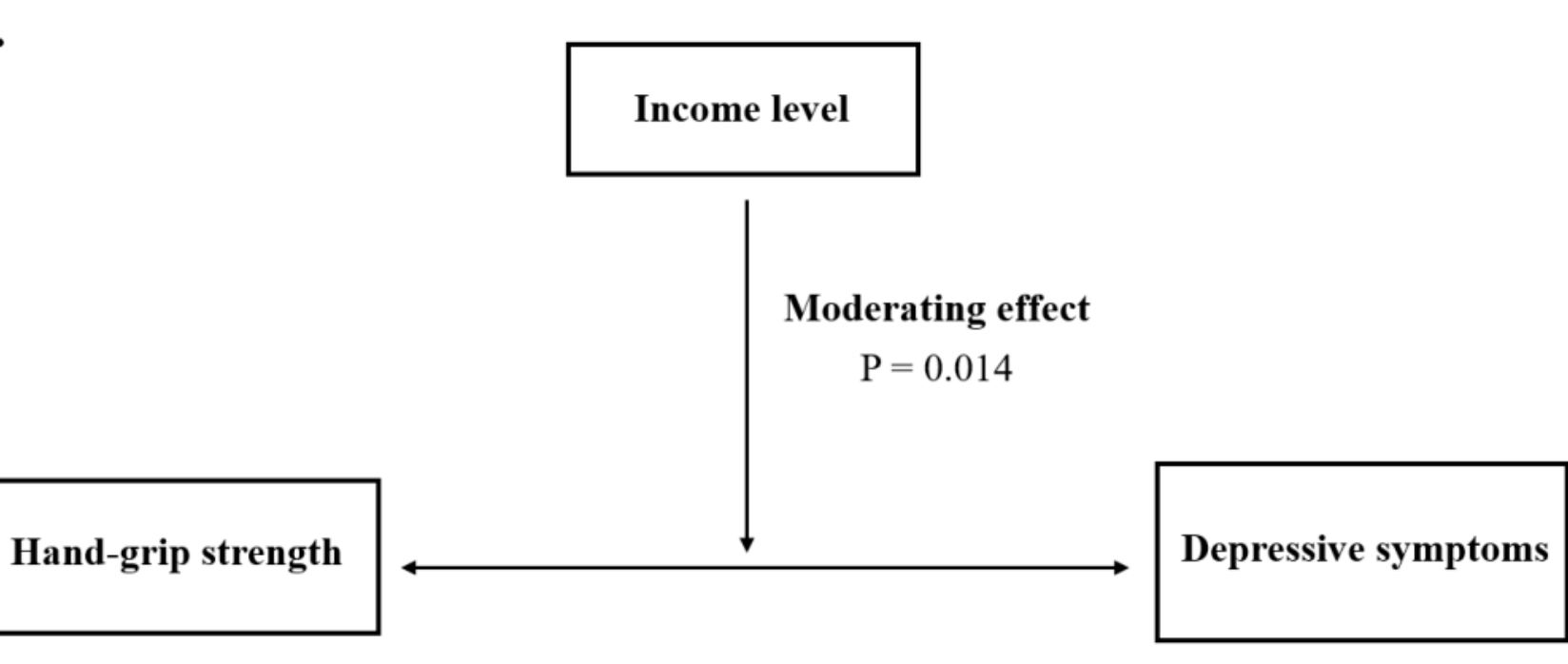

B.

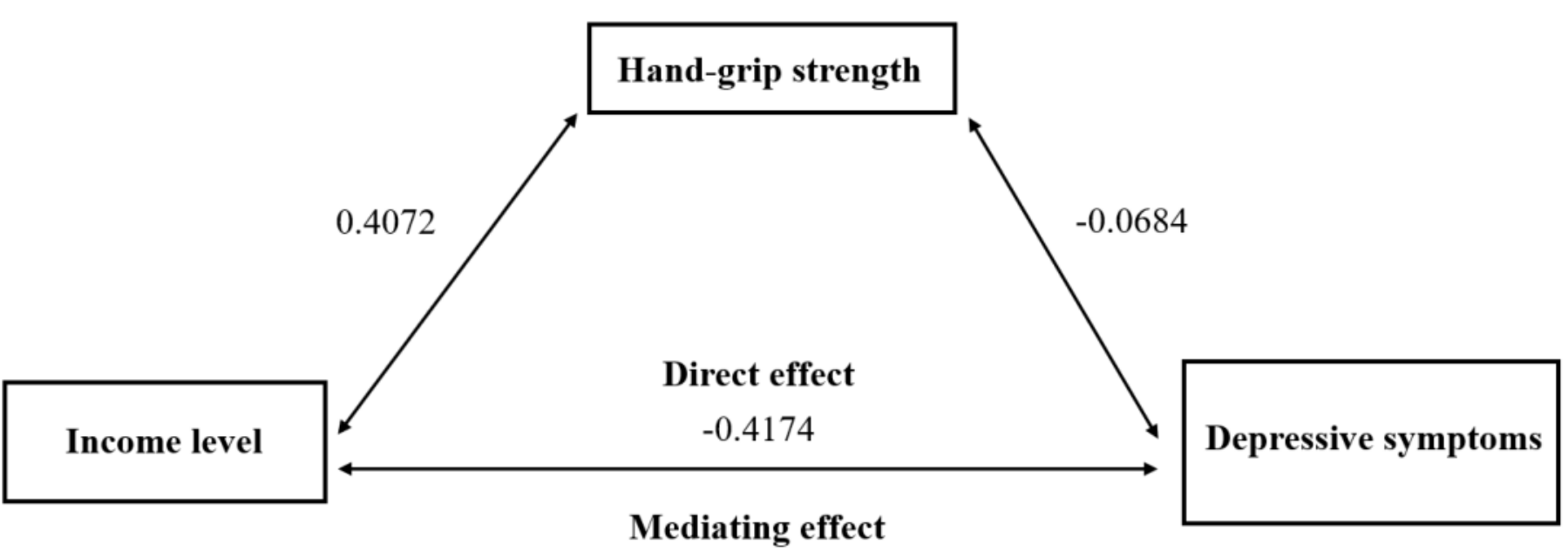

$-0.0278$

Figure 1. Moderation (Panel A) and mediation models (Panel B) of household income level, hand-grip strength (HGS), and depressive symptoms in older adults.

Conclusion: In summary, we explored the complex relationships between HGS, SES, and depressive symptoms using a nationally representative sample of the older Korean population. Our findings indicate that socioeconomically deprived older people may be more vulnerable to the detrimental effects of dynapenia-which reflects poor physical performance-on depressive symptoms, or could be more susceptible to the negative impacts of depressive symptoms on muscle strength. Policy makers and clinicians should be aware of muscle strength and income level during risk assessment for depression onset among older adults. We hope a future longitudinal study can disentangle this complex issue. 